\title{
The BAIC BJEV view of autonomous driving in China
}

Marcus Hafkemeyer

Beijing Electrical Vehicle Co., Ltd (BAIC BJEV)

This manuscript is not available according to publishing restriction. Thank you for your understanding. 